\title{
SpoIIIE strips proteins off the DNA during chromosome translocation
}

\author{
Kathleen A. Marquis, ${ }^{1}$ Briana M. Burton, ${ }^{2}$ Marcelo Nollmann, ${ }^{3}$ Jerod L. Ptacin, ${ }^{3}$ \\ Carlos Bustamante, ${ }^{3}$ Sigal Ben-Yehuda, ${ }^{4}$ and David Z. Rudner ${ }^{1,5}$ \\ ${ }^{1}$ Department of Microbiology and Molecular Genetics, Harvard Medical School, Boston, Massachusetts 02115, USA; \\ ${ }^{2}$ Department of Cell Biology, Harvard Medical School, Boston, Massachusetts 02115, USA; ${ }^{3}$ Department of Molecular and \\ Cell Biology, University of California, Berkeley, California 94720, USA; ${ }^{4}$ Department of Molecular Biology, The Hebrew \\ University of Jerusalem, Jerusalem 91120, Israel
}

The FtsK/SpoIIIE family of DNA transporters are responsible for translocating missegregated chromosomes after the completion of cell division. An extreme example of this post-cytokinetic DNA segregation occurs during spore formation in the bacterium Bacillus subtilis, where SpoIIIE pumps three-quarters of the chromosome (>3 megabases) into one of the two daughter cells. Here, we investigate the fate of the proteins associated with the translocated DNA. Taking advantage of several unique features of Bacillus sporulation, we demonstrate that RNA polymerase, transcription factors, and chromosome remodeling proteins are stripped off the DNA during translocation of the chromosome into the forespore compartment. Furthermore, we show that in vitro the soluble ATPase domain of SpoIIIE can displace RNA polymerase bound to DNA, suggesting that SpoIIIE alone is capable of this wire-stripping activity. Our data suggest that the bulk of the forespore chromosome is translocated naked into the forespore compartment. We propose that the translocation-stripping activity of SpoIIIE plays a key role in reprogramming developmental gene expression in the forespore.

[Keywords: Chromosome segregation; chromosome translocation; sporulation; FtsK; AAA+ ATPase]

Supplemental material is available at http://www.genesdev.org.

Received April 11, 2008; revised version accepted May 9, 2008.

Faithful chromosome segregation is a critical event in the cell division cycle of all organisms. In bacteria, the replicated chromosomes are not always completely segregated prior to cytokinesis. In these situations, an ATPase of the FtsK/SpoIIIE family is required to translocate the unsegregated DNA into one of the two daughter cells (Errington et al. 2001). These DNA transporters are polytopic membrane proteins with cytoplasmic ATPase domains. In single molecule experiments, the soluble ATPase domains (the motor domain) of these proteins can translocate along double-stranded DNA at a rate of $\sim 4 \mathrm{kbp} / \mathrm{sec}$ (Saleh et al. 2004; Pease et al. 2005; Ptacin et al. 2008). The crystal structure of the cytoplasmic motor domain of FtsK indicates that it assembles into hexameric rings that are large enough to accommodate dsDNA (Massey et al. 2006). Visualization of the soluble FtsK motor domain bound to DNA by electron microscopy supports the idea that dsDNA passes through the central pore (Aussel et al. 2002; Massey et al. 2006).

Spore formation in Bacillus subtilis provides an ideal system in which to investigate how this family of trans-

${ }^{5}$ Corresponding author.

E-MAIL rudner@hms.harvard.edu; FAX (617) 738-7664.

Article is online at http://www.genesdev.org/cgi/doi/10.1101/gad.1684008. porters functions in vivo. In B. subtilis, the DNA translocase SpoIIIE is involved in transporting missegregated DNA during vegetative growth (Sharpe and Errington 1995; Britton and Grossman 1999) and plays an essential role in chromosome segregation during the process of sporulation (Wu and Errington 1994). In response to nutrient starvation, $B$. subtilis enters a developmental pathway that culminates in the formation of a stressresistant cell type known as a spore (Stragier and Losick 1996; Errington 2003; Hilbert and Piggot 2004). Upon entry into sporulation, an asymmetrically positioned septum divides the developing cell into a large cell (the mother cell) and a small cell (the prospective spore or forespore) (Fig. 1A). Initially, the two cells lie side by side, but shortly after polar division the mother cell membranes migrate around the forespore generating a cell within a cell. At this stage, the mother nurtures the forespore, packaging it in a protective protein coat while the forespore prepares for dormancy. Once the spore is fully mature, it is released into the environment through lysis of the mother cell. Throughout this process, the forespore and mother cell follow different developmental programs of gene expression that are governed by a cascade of transcription factors that are activated in a temporally and spatially restricted fashion. 
A
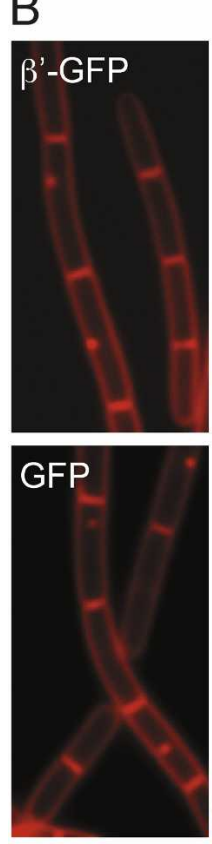

membranes

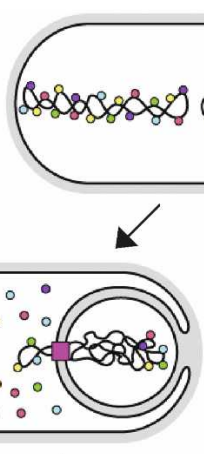

stripped
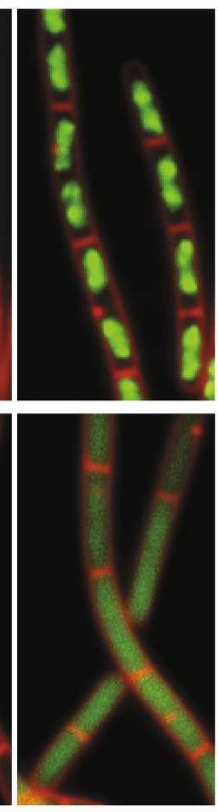

GFP

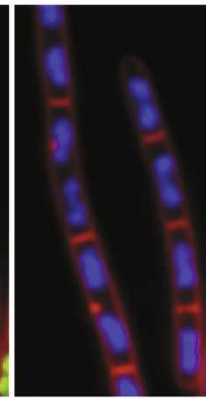

translocated

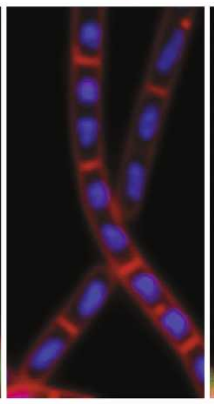

DNA
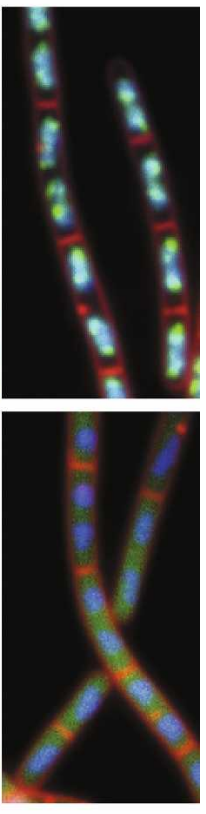

merge

Figure 1. Two models for the fate of DNA-binding proteins during chromosome translocation. (A) In the first model DNAassociated proteins (colored circles) are stripped off the forespore chromosome during DNA transport. In the second model these proteins are translocated into the forespore along with the DNA. The SpoIIIE translocase (pink rectangle) is shown. The origins of replication are located near the cell poles and the termini are close to midcell. $(B)$ RNA polymerase colocalizes with the chromosome (nucleoid) inside the cell. Fluorescent micrographs of vegetatively growing $B$. subtilis cells harboring a functional $\beta^{\prime}$-GFP fusion (strain BDR1499). $\beta^{\prime}$-GFP colocalizes with the DAPI-stained nucleoid. For comparison, a strain (BDR1778) producing free GFP localizes as a diffuse haze throughout the cytoplasm. A similar nucleoid-associated localization was observed for Hbsu-GFP (data not shown). Higher resolution versions of the figures are available in Supplemental Material.

Upon the initiation of sporulation, the replicated chromosomes are remodeled into an elongated structure that extends from one cell pole to the other. This serpentine structure is called the axial filament (Ryter et al. 1966; Ben-Yehuda et al. 2003b). The origin regions are located at the extreme cell poles of the axial filament while the

termini reside at midcell (Lin et al. 1997; Webb et al. 1997; Wu and Errington 1998). As a result of axial filament formation, the asymmetric division plane traps approximately one quarter of the forespore chromosome (the origin-proximal region) in the small forespore compartment (Wu and Errington 1998; N. Sullivan and D.Z. Rudner, unpubl.). After the completion of cytokinesis, SpoIIIE assembles on the DNA trapped in the septum (Sharp and Pogliano 1999; Ben-Yehuda et al. 2003a) and is responsible for pumping the remaining three quarters of the chromosome ( $>3$ megabases) from the mother cell into the forespore (Wu and Errington 1994). Thus, in this unusual cell cycle cytokinesis precedes DNA segregation.

An outstanding question related to the FtsK/SpoIIIE family of transporters is what happens to the proteins associated with the DNA during chromosome translocation (Fig. 1A). Are these proteins transported along with the DNA across the division septum? Or are they stripped off during translocation? In the case of sporulation, either answer could have profound implications for developmental gene expression. Since DNA-binding proteins are predominantly associated with the chromosome and not free in the cytoplasm (Fig. 1B), if these proteins are translocated along with the DNA, their concentration in the small forespore compartment could increase significantly. Conversely, if these proteins are stripped off the DNA during translocation, this could help direct developmental gene expression by resetting epigenetic nucleoprotein structures and/or by excluding vegetative transcription factors from the developing spore.

No study has addressed what happens to proteins associated with the chromosome during DNA transport across the division septum. The pore size of the FtsK hexameric ring in the crystal structure is $\sim 30 \AA$. Doublestranded DNA could easily be threaded through this annulus, but proteins associated with the DNA might not be accommodated. In vitro experiments with the cytoplasmic motor domains of FtsK and SpoIIIE indicate that these enzymes can displace ssDNA when it encounters a DNA triplex (Bigot et al. 2005; Levy et al. 2005; Ptacin et al. 2008), suggesting that DNA-binding proteins might also be stripped off. However, some DNA-binding proteins have very high affinity for their cognate binding sites. In fact, in some instances these interactions are sufficient to impede the movement of a translocating complex as strong as the replisome (Possoz et al. 2006). The biophysical data presently available for either FtsK or SpoIIIE do not allow us to estimate whether these enzymes could displace proteins tightly bound to DNA. On the other hand, studies of several other AAA+ ATPases have revealed surprisingly elastic substrate accommodation. Hexamer to heptamer transitions resulting in larger pore sizes have been described for bacteriophage T7 primase (Crampton et al. 2006). Moreover, recent experiments analyzing protein translocation into the AAA + ClpXP proteosome revealed that this caged protease with an annular entry port can translocate and degrade branched substrates (Bolon et al. 2004). Thus, 
the channels of these translocation machines might be more flexible than their crystal structures suggest.

Taking advantage of unique features of $B$. subtilis sporulation, we analyzed the fate of proteins associated with the DNA during chromosome translocation in vivo. We demonstrate that RNA polymerase, chromosome remodeling proteins, and transcription factors are stripped off the chromosome during DNA transport. In particular, we show that fluorescent repressor proteins (TetR-GFP) bound to an array of operators (tetO) are efficiently removed from the forespore chromosome during translocation. By contrast, TetR-GFP bound to a tet $O$ array is sufficient to block progression of the replisome during chromosome replication. These results are consistent with the observation that the FtsK/SpoIIIE translocase is a more powerful motor than the replisome. In vitro experiments using the soluble motor domain of SpoIIIE indicate that SpoIIIE can displace RNA polymerase from transcription initiation complexes and stalled elongation complexes. Finally, we present evidence that the "wire-stripping" activity of SpoIIIE plays a role in reprogramming developmental gene expression in the forespore during sporulation.

\section{Results}

\section{Proteins are stripped off the chromosome} during translocation in a disporic mutant

To investigate the fate of DNA-binding proteins during chromosome translocation, we took advantage of a sporulation mutant that forms septa at both poles of the cell. These abortively disporic cells generate two forespores separated by a mother cell compartment (Fig. 2A; Stragier and Losick 1996). SpoIIIE assembles at both polar septa, and the replicated chromosomes are pumped into the two forespores, leaving the mother cell devoid of DNA (Wu and Errington 1994). Since DNA-binding proteins are predominantly associated with the chromosome (Figs. 1B, 2B), if these proteins are translocated along with the DNA, the mother cell should be depleted of DNA-binding proteins. On the other hand, if proteins associated with the DNA are stripped off, then the proteins initially bound to the chromosome in the mother cell should be released into the mother cell cytoplasm during DNA transport.

For these experiments we used a functional fusion of the $\beta^{\prime}$ subunit of RNA polymerase to GFP $(r p o C-g f p)$. As we observed for vegetative cells (Fig. 1B), RNA polymerase specifically colocalized with the replicated chromosomes during sporulation in wild-type cells (Fig. 2B). In the disporic mutant, when DNA translocation was not yet complete, RNA polymerase colocalized with the DNA in both the mother cell and forespore compartments (Fig. 2B, yellow caret). However, in those cells in which chromosome translocation was complete, as judged by loss of DAPI signal (Fig. 2B, white carets), RNA polymerase was observed as a diffuse haze in the mother cell. Comparison of total $\beta^{\prime}$-GFP fluorescence intensity in the mother cell before and after DNA translocation indicates that $85 \%-90 \%$ of the RNA polymerase ini-
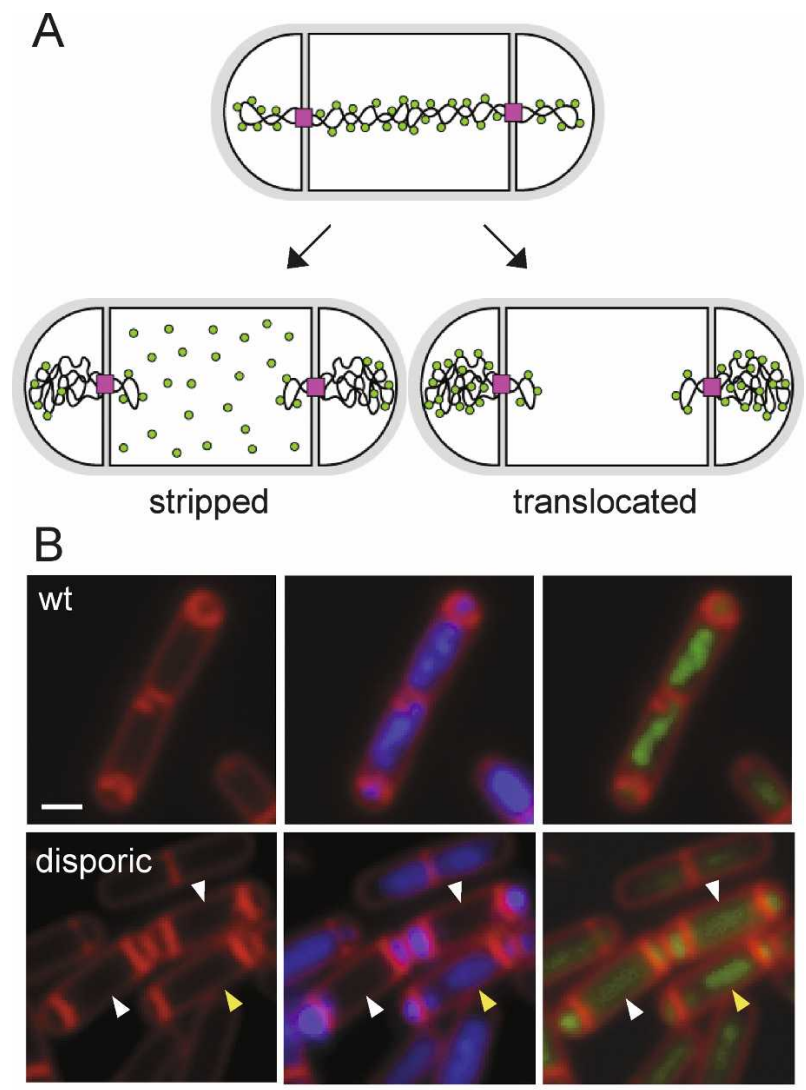

membranes

DNA

GFP

Figure 2. RNA polymerase is stripped off the DNA during chromosome translocation. (A) Schematic diagram of the experiment in $B$ with the two possible outcomes. An abortively disporic mutant is shown with RNA polymerase (green balls) associated with the replicated chromosomes. If RNA polymerase is stripped off the DNA, the fluorescent fusion protein will be present as a diffuse haze in the mother cell cytoplasm. If RNA polymersase is translocated along with the DNA, the mother cell will be devoid of fluorescent signal. The SpoIIIE translocase (pink square) is shown. (B) $\beta^{\prime}$-GFP is stripped off the chromosome during translocation into the forespore. Fluorescent micrographs of sporulating $B$. subtilis cells harboring a functional $\beta^{\prime}$-GFP fusion. The top panels show the colocalization of $\beta^{\prime}$-GFP with the nucleoid in wild-type sporulating cells (strain BDR1499). The bottom panels show $\beta^{\prime}$-GFP in a disporic mutant (strain BDR1522). An abortively disporic sporangium that has not yet completed transport of the chromosomes into the forespore compartments is shown (yellow caret). In this cell $\beta^{\prime}$-GFP colocalizes with the DNA that is still present in the mother cell compartment. Two abortively disporic sporangia that have finished DNA translocation are indicated (white carets). In these cells there is no DAPI signal in the mother cell and $\beta^{\prime}$-GFP is visible as a diffuse haze in this compartment. The signal appears weaker because the protein is no longer concentrated on the DNA. Quantitation of fluorescent intensity revealed that $85 \%-90 \%$ of the mother cell signal remains after DNA transport. Similar results were obtained with Hbsu-GFP and RacA-GFP (data not shown). Bar, $1 \mu \mathrm{m}$.

tially present in the mother cell was retained in the mother cell compartment. Since proteins are unable to diffuse between the two compartments $\mathrm{Wu}$ and Errington 1997), we interpret these results to indicate that 
RNA polymerase was stripped off the chromosomes during transport. The gene fusion was inserted at its endogenous locus ( $11^{\circ}$ relative to the origin of replication), and this region is trapped in the forespore compartment at the time of polar septation. Thus, new transcription of rpoC-gfp could only occur in the forespores during DNA translocation. In fact, the signal in the forespores appeared brighter at late time points (data not shown). We also investigated the fate of two additional DNA-binding proteins during chromosome translocation: the developmentally regulated chromosome remodeling protein RacA (RacA-GFP) (Ben-Yehuda et al. 2003b) and the small histone-like protein Hbs (Hbs-GFP) (Kohler and Marahiel 1997). In both cases a diffuse fluorescent signal similar to $\beta^{\prime}$-GFP was observed in the mother cell compartment when DNA transport was complete (data not shown). Altogether, these data are consistent with the idea that DNA-binding proteins are stripped off the DNA during chromosome translocation.

\section{TetR-GFP is stripped off tetO arrays during translocation}

In the experiments described above, we visualized DNAbinding proteins bound specifically and nonspecifically throughout the genome. To investigate the fate of a transcription factor bound site specifically, we used the TetR repressor protein fused to GFP (TetR-GFP) and an array of tet operators (tetO) (Michaelis et al. 1997). This repressor-operator system has been used extensively to visualize the subcellular location of specific regions of the chromosome in bacteria and eukaryotic cells (Michaelis et al. 1997; Webb et al. 1997; Viollier et al. 2004; Wang et al. 2005). When the tet $O$ array was placed near the origin of replication and the TetR-GFP fusion was synthesized prior to polar division, two foci (one for each chromosome) could be easily visualized (Supplemental Fig. S1B). As was found previously (Webb et al. 1997), one of the foci was present in the mother cell and the other in the forespore (Supplemental Fig. S1B).

To investigate the fate of TetR-GFP bound to the tet $O$ array during chromosome translocation, we placed the tetR-gfp fusion under the control of a mother cell-specific promoter $\left(\mathrm{P}_{\text {spoIID }}\right)$ such that the fluorescent repressor protein would be synthesized exclusively in the mother cell compartment (Fig. 3A). In addition, the tet $O$ array was inserted at a site $\left(130^{\circ}\right)$ that is present in the mother cell at the time of polar septation and must be translocated into the forespore (Fig. 3A). Accordingly, at early time points after asymmetric division, two foci (one for each chromosome) should be present in the mother cell compartment. We could then assess the fate of the TetR-GFP focus associated with the forespore chromosome at late time points once DNA translocation was complete (Fig. 3A). Unfortunately, using this experimental setup we could only detect one fluorescent focus in the mother cell (and none in the forespore) at early time points (data not shown). Whereas the SpoIIIE initiates DNA transport immediately after division is complete, the first mother cell-specific transcription factor
$\left(\sigma^{\mathrm{E}}\right)$ is activated only after a short delay (Zhang et al. 1996; Zupancic et al. 2001). Thus, we suspect that the terminus region of the chromosome (including the tet $O$ array) is transported into the forespore before TetR-GFP accumulates (or folds or matures) to significant levels. To circumvent this problem, we took advantage of a newly discovered SpoIIIE mutant (SpoIIIE $\left.{ }^{\mathrm{D} 584 \mathrm{~A}}\right)$ whose rate of DNA transport is reduced by 2.5 -fold (Burton et al. 2007). Using this mutant background, we could detect two TetR-GFP foci in the mother cell in most $179 \%$, $n=297$ ) of the sporulating cells at early time points (Fig. 3B). Consistent with the idea that TetR-GFP is stripped off the DNA, at late time points, when chromosome transport was complete, we could detect a single bright focus in the mother cell and no focus in the forespore in virtually all $(98 \%, n=324)$ of the cells (Fig. 3B). Similar results were obtained when tet $O$ arrays were placed at $100^{\circ}$ or near the terminus at $162^{\circ}$ (data not shown). As reported previously, arrays close to the terminus are not easily resolved prior to translocation (Bogush et al. 2007). However, moments before translocation of the $162^{\circ}$ region, we could detect two foci in the mother cell compartment of some cells (Supplemental Fig. S2). Importantly, we never observed foci in the forespores. Altogether, these results indicate that DNA-binding proteins are stripped off the DNA during chromosome translocation.

Similar results were obtained in a strain containing wild-type SpoIIIE. In this strain TetR-GFP was synthesized at the onset of sporulation in the predivisional cell (using the $\mathrm{P}_{\text {spoIIE }}$ promoter), and upon polar septation its synthesis was shut down in the forespore but persisted in the mother cell compartment (Supplemental Fig. S1A; Fujita and Losick 2003). In this background, when the tet $O$ array was present at $-91^{\circ}$, two bright foci could be seen in the mother cell upon polar division (Supplemental Fig. S1B). Once translocation was complete, a bright focus was visible in the mother cell and a faint focus could be detected in the forespore. We interpret these results to mean that the mother cell TetR-GFP was stripped off during DNA translocation and the low levels of TetR-GFP present in the forespore bound these sites upon their arrival in the forespore compartment. Consistent with this idea, when the tet $O$ array was placed near the origin $\left(-7^{\circ}\right)$, such that at the time of polar division the array was already present in the forespore compartment, the mother cell and forespore foci remained at equal intensity after DNA translocation (Supplemental Fig. S1B).

Repressors bound to arrays of operators have been found to block the progression of the replisome in Escherichia coli (Possoz et al. 2006). We observed a similar replication roadblock in $B$. subtilis (Supplemental Fig. S4). In the experiments described above TetR-GFP was synthesized after completion of DNA replication but prior to DNA transport. We wondered whether TetRGFP bound to the tet $O$ array might impede or slow down DNA translocation and therefore cause a sporulation defect. To test this, we analyzed sporulation efficiency in the strains described above. In support of the idea that SpoIIIE efficiently strips these proteins off the DNA, in 
all strains tested the sporulation efficiency was similar to wild type (Supplemental Table S1). These results are consistent with reports that FtsK/SpoIIIE translocases are stronger motors than the replisome (Wuite et al. 2000; Pease et al. 2005) and altogether these experiments support the view that the forespore chromosome arrives naked in the developing spore.

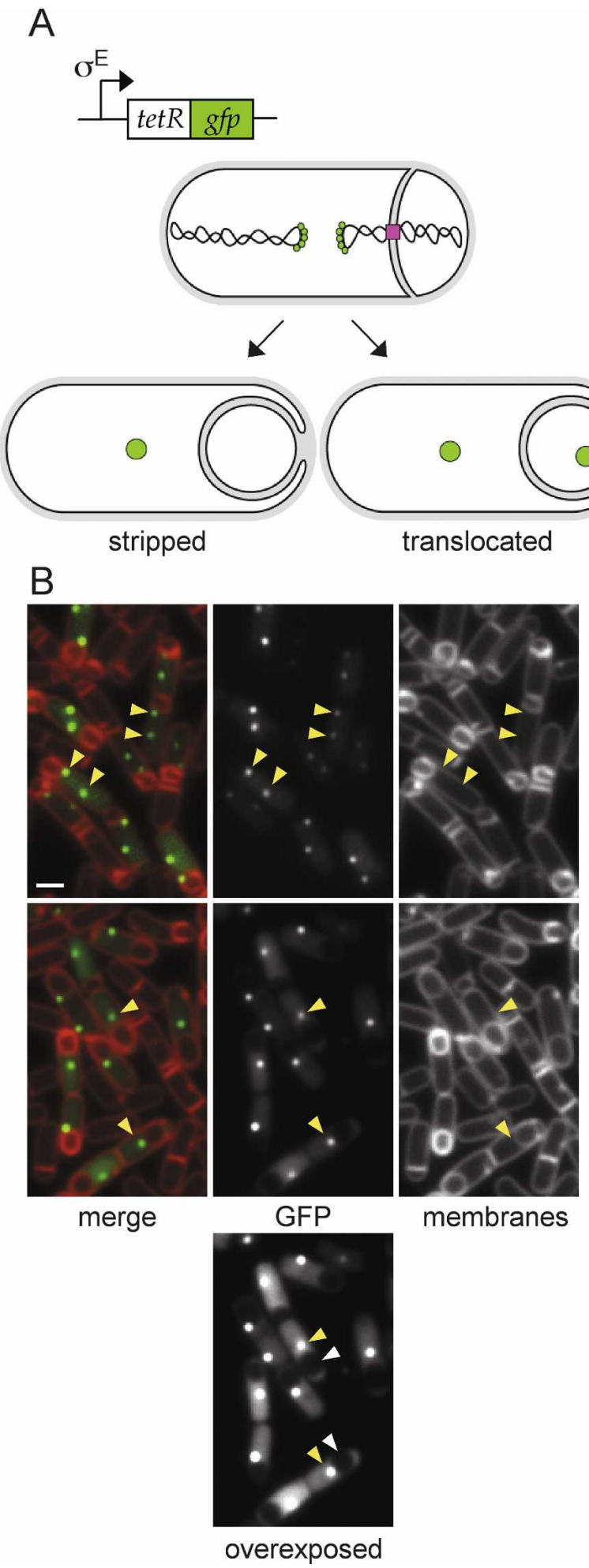

The ATPase domain of SpoIIIE can displace RNA polymerase bound to DNA in vitro

To investigate whether SpoIIIE is responsible for clearing proteins from the DNA during translocation, we analyzed the ability of SpoIIIE to displace RNA polymerase bound to DNA in vitro. For these experiments we used the soluble motor domain of SpoIIIE, which has been shown to translocate along DNA in vitro (Bath et al. 2000; Ptacin et al. 2008). E. coli RNA polymerase containing $\sigma^{70}$ was assembled on a fluorescently labeled DNA substrate containing the $\lambda_{\mathrm{PR}}$ promoter. Complexes were formed in the absence of ribonucleotides to generate an initiation complex or in the presence of three of the four ribonucleotides to create a stalled elongation complex (see the Materials and Methods). Both complexes were stable in a gel mobility shift assay (Fig. 4; Supplemental Fig. S3). When these complexes were incubated with either the motor domain of SpoIIIE or high concentrations of ATP, RNA polymerase remained stably associated with the DNA (Fig. 4A; Supplemental Fig. S3). However, when both SpoIIIE and ATP were present, RNA polymerase was displaced from the DNA substrate (Fig. 4A; Supplemental Fig. S3). To confirm that the displaced complex was a stalled elongation complex, we followed the fate of the RNA transcript by generating stalled complexes as described above but in the presence of $\alpha^{32}$ P-GTP (see the Materials and Methods). This ternary complex composed of RNA polymerase, a radioactive transcript, and the substrate DNA was stable in a gel mobility shift assay and remained intact in the presence of either the motor domain of SpoIIIE or high concentrations of ATP (Fig. 4B). Addition of both SpoIIIE and ATP efficiently dissociated the ternary complex (Fig. 4B). We interpret this to mean that the ATP-dependent motor

Figure 3. Transcriptional regulators are stripped off the DNA during translocation. (A) Schematic diagram of the experiment in $B$ with the two possible outcomes. An array of tet $O$ operators was inserted at a position near the teriminus $\left(130^{\circ}\right)$ that is always present in the mother cell at the time of polar septation. In this strain, the tetR-gfp fusion was placed under the control of a mother cell-specific ( $\sigma^{\mathrm{E}}$-dependent) promoter. Finally, this strain has a mutant SpoIIIE translocase (pink square) that pumps the chromosome 2.5-fold slower than wild type (Burton et al. 2007). Thus, at early time points after $\sigma^{\mathrm{E}}$ becomes active in the mother cell, two TetR-GFP foci should be visible in the mother cell. If TetR-GFP is stripped off the DNA, one of these two foci will be missing when chromosome translocation is complete. If TetR-GFP is translocated with the chromosome, when DNA transport is complete, one TetR-GFP focus will be visible in the forespore and one in the mother cell. $(B)$ TetR-GFP bound to the tet $O$ array is stripped off the chromosome during translocation. Fluorescence micrographs of sporulating B. subtilis cells (strain BKM1016) at early (hour 2) and late (hour 3) time points. At hour 2, prior to the completion of DNA translocation, two fluorescent foci are visible in the mother cell (yellow carets). At hour 3 , when chromosome translocation is complete in most cells, a single focus is visible in the mother cell (yellow caret) and no detectable foci are seen in the forespore (white caret). No TetRGFP foci are visible in the forespore when the micrograph is rescaled (overexposed) to reveal weak signals. Bar, $1 \mu \mathrm{m}$. 


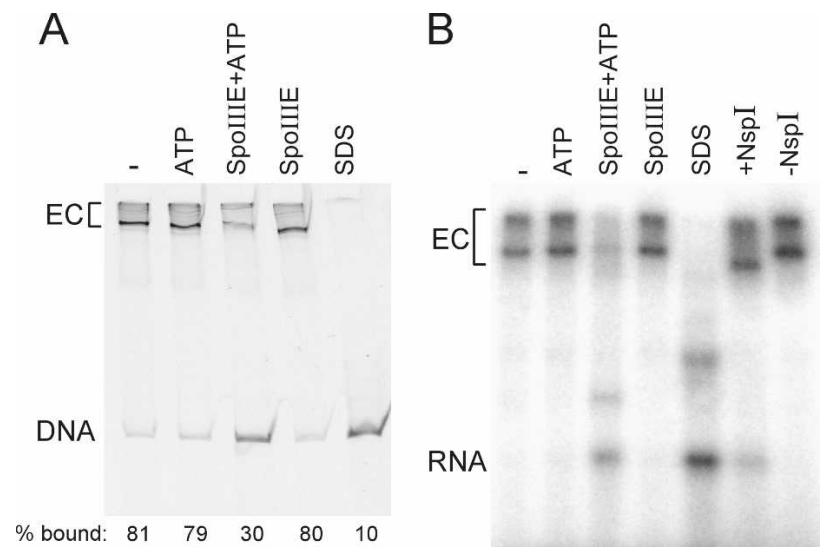

Figure 4. The soluble motor domain of SpoIIIE can displace a stalled transcription elongation complex in vitro. (A) E. coli RNA polymerase was incubated with a fluorescently labeled DNA substrate containing the $\lambda_{\mathrm{PR}}$ promoter. The reaction contained $25 \mu \mathrm{M}$ UTP, GTP, and ATP but no CTP to generate a stalled elongation complex (EC). The stalled complexes were then incubated with $3 \mathrm{mM}$ ATP, $420 \mathrm{nM}$ SpoIIIE, and $3 \mathrm{mM}$ ATP, 420 nM SpoIIIE, or $5 \%$ SDS and analyzed by gel mobility shift on a native polyacrylamide gel. The fraction of RNA polymerase present in the elongation complex is indicated below the gel. $(B)$ E. coli RNA polymerase was incubated with the $\lambda_{\mathrm{PR}}$ promoter and $2.5 \mu \mathrm{M}$ UTP, GTP, ATP and $0.7 \mu \mathrm{M} \alpha^{32} \mathrm{P}$-GTP but no CTP to generate a stalled elongation complex with a short radiolabeled transcript. The stalled complex was incubated with $3 \mathrm{mM}$ ATP, $200 \mathrm{nM}$ SpoIIIE, and $3 \mathrm{mM}$ ATP, $200 \mathrm{nM}$ SpoIIIE, or $5 \%$ SDS and the reactions were analzyed on a native polyacrylamide gel followed by autoradiography. Dissassembly of the complex is evidenced by release of the labeled transcript. The stalled complex was confirmed by the ability of NspI to cut the DNA substrate $(+\mathrm{NspI})$ within the promoter region, resulting in a faster migrating complex.

activity of SpoIIIE stripped RNA polymerase from the DNA, resulting in release of the labeled transcript (Andrews and Richardson 1985). Taken together, these results indicate that the motor domain of SpoIIIE can displace RNA polymerase during transcription initiation and in the context of a stalled elongation complex. Dissociation of transcription elongation complexes has been described previously for the E. coli transcription repair coupling factor (Mfd) (Selby and Sancar 1993). Although it is possible that an analogous protein participates in displacing RNA polymerase in vivo, our in vitro data suggest that SpoIIIE on its own is capable of removing these complexes during DNA transport.

\section{Vegetative transcription is down-regulated in the forespore}

We wondered whether stripping of RNA polymerase and its associated vegetative (housekeeping) $\sigma$ factor $\left(\sigma^{\mathrm{A}}\right)$ might result in lower transcription from $\sigma^{\mathrm{A}}$-dependent promoters in the forespore compared to the mother cell. Attempts to monitor expression of vegetative promoters fused to $g f p$ during sporulation were not informative due to the relative stability of GFP and its production during vegetative growth prior to polar division (data not shown). To circumvent these problems, we fused the fluorescent reporter to a xylose-inducible $\sigma^{\mathrm{A}}$-dependent promoter $\left(\mathrm{P}_{x y l A}\right)$ (Fig. 5A). Accordingly, we could induce $\sigma^{\mathrm{A}}$-dependent gene expression during chromosome translocation and monitor GFP levels shortly after its completion. The $\mathrm{P}_{x y l A}-g f p$ reporter was inserted at a position $\left(28^{\circ}\right)$ in the chromosome that is trapped in the forespore at the time of polar division (Fig. 5A). Therefore, at the time of induction, both the mother cell and forespore contain a copy of the reporter. Synchronous sporulation was induced and, when $\sim 40 \%$ of the cells had a polar septum (hour 1) (Fig. 5A) and were just beginning DNA transport, xylose was added to the culture. Thirty minutes later (hour 1.5) GFP was visualized by fluorescence microscopy. In those cells that had just

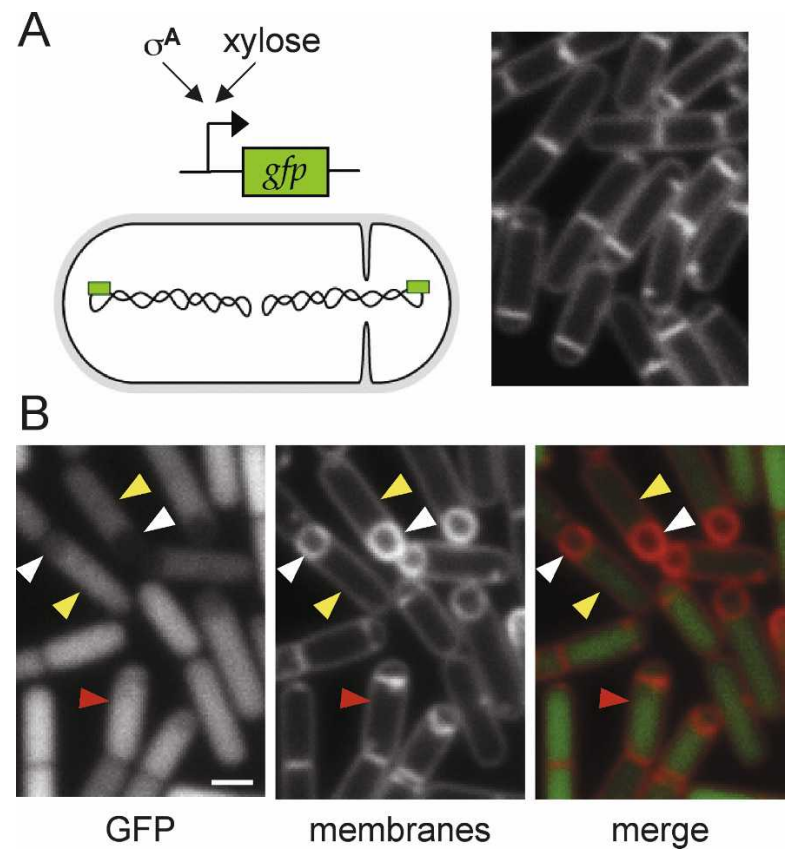

Figure 5. Vegetative gene expression is down-regulated in the forespore after chromosome translocation. As a result of the wire-stripping activity of SpoIIIE, much of $\sigma^{\mathrm{A}}$-associated RNA polymerase will be stripped off the forespore chromosome during translocation. One prediction of this model is that $\sigma^{\mathrm{A}}$-directed transcription will be reduced in the forespore compared to the mother cell. $(A)$ The xylose-inducible $\sigma^{\mathrm{A}}$ promoter $\mathrm{P}_{x y l A}$ was fused to $g f p$ and placed at an original-proximal position of the chromosome (strain BDR734), and synchronous sporulation was induced in the absence of xylose. When $\sim 40 \%$ of the sporulating cells had generated a polar septum (hour 1) xylose was added (20 $\mathrm{mM}$ final concentration) to induce transcription from the $\mathrm{P}_{x y l A}$ promoter. $(B)$ GFP fluoresence was visualized $30 \mathrm{~min}$ later (hour 1.5). In sporulating cells in which DNA translocation had initiated or was complete, GFP fluorescence was reduced in the forespore (white caret) compared to the mother cell (yellow caret). In cells that had just assembled an asymmetric septum, the signal in both compartments was similar (red caret). Cellto-cell variability in the intensity of the GFP signal is due to the differences in stages of sporulation at the time of xylose addition. Bar, $1 \mu \mathrm{m}$. 
formed a polar septum, the GFP signal was similar in both the mother cell and forespore compartments (red caret in Fig. 5B). However, in cells that had been translocating their DNA during the induction period, there was less GFP signal in the forespore than the mother cell (yellow and white carets in Fig. 5B). Li and Piggot (2001) have shown previously that $\sigma^{\mathrm{A}}$ continues to be active in the forespore both before and after completion of engulfment. Consequently, some $\sigma^{\mathrm{A}}$ activity in the forespore was expected. There was some cell-to-cell variability in the intensity of GFP signal due to differences in the developmental stage of the sporulating cells at the time of xylose addition (i.e., whether or not polar septation had occurred). Accordingly, the relevant comparison is between mother cell and forespore in the same sporangia. It is not known whether xylose continues to be transported into the forespore once engulfment is complete. It is therefore possible that the reduced expression from this promoter could be due, in part, to lower concentrations of the inducer in the spore compartment. However, clear differences in the levels of GFP in the mother cell and forespore could be observed at early stages of engulfment when both cells were in direct contact with the medium (Fig. 5B). These results suggest that $\sigma^{\mathrm{A}}$-dependent transcription is lower in the forespore than the mother cell and are consistent with the idea that stripping $\sigma^{\mathrm{A}}$ from the forespore chromosome facilitates the down-regulation of housekeeping gene expression in the forespore compartment.

\section{Discussion}

We show that RNA polymerase, a transcriptional regulator, and chromosome packaging/remodeling proteins are stripped off the chromosome during DNA transport. Moreover, our in vitro analysis is consistent with the idea that SpoIIIE alone is responsible for this wire-stripping activity. If, as our data suggest, all proteins are stripped off the forespore chromosome during DNA transport, then $\sim 75 \%$ of the forespore genome ( $>3 \mathrm{Mb}$ of DNA) arrives in the forespore compartment naked ready to receive and carry out the dictates of the developing spore. We hypothesize that stripping plays a key role in reprogramming developmental gene expression by resetting epigenetic nucleoprotein structures and reducing the concentration of vegetative transcription factors in the developing spore.

Our observation that $\sigma^{\mathrm{A}}$-dependent gene expression is reduced in the forespore compartment compared to the mother cell is consistent with the idea that stripping down-regulates vegetative gene expression in the forespore. The down-regulation of housekeeping genes could allow the developing spore to focus its attention on the preparation for dormancy. We note that our experiments cannot distinguish between reduced $\sigma^{\mathrm{A}}$-dependent gene expression due to stripping of $\sigma^{\mathrm{A}}$ and the possibility that the activation of $\sigma^{\mathrm{F}}$ in the forespore results in competition for core RNA polymerase (Ju et al. 1999; Grigorova et al. 2006) and therefore a reduced $\sigma^{\mathrm{A}}$ holoenzyme pool. However, these models are not mutually exclusive. $\sigma^{\mathrm{F}}$ is made in the predivisional cell but is held inactive in the cytoplasm by the anti-sigma factor SpoIIAB (Stragier and Losick 1996; Rudner and Losick 2001; Hilbert and Piggot 2004). When cytokinesis is complete, $\sigma^{\mathrm{F}}$ is released from SpoIIAB in the forespore compartment where it associates with core RNA polymerase. Stripping $\sigma^{\mathrm{A}}$ off the forespore chromosome likely helps shift the balance in favor of $\sigma^{\mathrm{F}}$ and thus helps lock the forespore into its developmental program (Lord et al. 1999).

It is noteworthy that Fujita and Losick (2003) have found that gene expression under the control of the transcription factor Spo0A (the master regulator for entry into the sporulation pathwayl is similarly lower in the forespore compared to the mother cell (Supplemental Fig. S1A). Moreover, these researchers showed that inappropriate expression of Spo0A-controlled genes in the forespore causes a 50-fold reduction in sporulation efficiency. It is possible, and we think likely, that stripping of Spo0A off the forespore chromosome during DNA transport contributes to the down-regulation of Spo0Acontrolled genes in the forespore compartment. In support of this idea, localization of Spo0A by immunofluorescence microscopy indicates that the levels of the transcription factor are lower in the forespore than the mother cell (Fujita and Losick 2003).

The removal of proteins from the forespore chromosome during transport likely plays an additional role in maintaining compartment-specific gene expression. The first mother cell-specific transcription factor $\sigma^{\mathrm{E}}$ becomes active shortly after polar division and prior to the completion of DNA transport (Zhang et al. 1996; Zupancic et al. 2001). Thus, removal of $\sigma^{\mathrm{E}}$ associated with the forespore chromosome would ensure that production of mother cell proteins under the control of $\sigma^{\mathrm{E}}$ is restricted to the mother cell compartment.

Another possible role for the stripping activity of SpoIIIE is in chromosome remodeling. As part of the preparation for dormancy, the forespore synthesizes a set of small acid-soluble proteins that compacts the chromosome into a doughnut-like structure (Pogliano et al. 1995) and confers resistance to DNA damaging agents (Setlow 1995). The stripping of vegetative proteins off the DNA could facilitate the remodeling of the chromosome into this dormant structure later in development.

Our data suggest that SpoIIIE can efficiently remove RNA polymerase from the chromosome during DNA transport. However, it is possible that head-on collisions between SpoIIIE and RNA polymerase might affect the rate of chromosome transport. Interestingly, the majority $(-75 \%)$ of the genes in the $B$. subtilis genome are transcribed toward the terminus and this unusually high bias is found in all endospore formers (Rocha 2004). It has been proposed that this coorientation with replisome movement has been selected to avoid collisions between RNA and DNA polymerases (Brewer 1988; French 1992). We wonder whether DNA translocation and the consequence of multiple head-on collisions between the transcription machinery and SpoIIIE might serve as an added selective pressure for coorientation.

In conclusion, we showed that DNA-binding proteins 
are actively removed from the chromosome during DNA translocation. Our in vitro studies suggest that SpoIIIE is sufficient for this wire-stripping activity; however, we do not exclude the possibility that other proteins also participate in removing tightly bound proteins during the translocation process. Finally, our data and those of others are consistent with the idea that the removal of DNA-binding proteins plays an important role in dictating developmental gene expression in the forespore. It remains unclear what impact, if any, the additional DNA-binding proteins removed from the forespore chromosome have on gene expression and chromosome dynamics in the mother cell. During vegetative growth, we suspect that SpoIIIE (and other FtsK/SpoIIIE family members) also removes proteins during the transport of missegregated DNA. In E. coli two recombination proteins, XerC and XerD, bind sequence elements (dif sites) near the replication terminus. These factors interact with FtsK, and this interaction is required for resolution of chromosome dimers (Aussel et al. 2002). The displacement of these proteins from the DNA prior to recombination would prevent dimer resolution. Thus, we hypothesize that interaction between XerC/D and FtsK prevents translocation and stripping until recombination is complete.

\section{Materials and methods}

\section{General methods}

All B. subtilis strains were derived from the prototrophic strain PY79 (Youngman et al. 1983) and are listed in Supplemental Table S2. E. coli strains were TG1 and DH5 $\alpha$. Sporulation was induced by resuspension at $37^{\circ} \mathrm{C}$ according to the method of Sterlini-Mandelstam (Harwood and Cutting 1990). Sporulation efficiency was determined in $36 \mathrm{~h}$ cultures as the total number of heat-resistant $\left(80^{\circ} \mathrm{C}\right.$ for $\left.20 \mathrm{~min}\right)$ colony forming units (CFU) compared with the number of wild-type heat-resistant CFU. To prevent selection for shrunken tet $O$ arrays, strains containing (tetO) $)_{120}$ and tetR-gfp were maintained in the presence of 40 $\mathrm{ng} / \mathrm{mL}$ anhydrotetracycline. The inducer was not present during experiments. Plasmids and oligonucleotide primers used in this study are listed in Supplemental Tables S3 and S4.

\section{Fluorescence microscopy}

Fluorescence microscopy was performed with an Olympus BX61 microscope as described (Doan et al. 2005). Fluorescent signals were visualized with a phase contrast objective UplanFLN 100x and captured with a monochrome CoolSnapHQ digital camera (Photometrics) using Metamorph software version 6.1 (Universal Imaging). Exposure times were typically 500 msec for GFP fusions (excitation: 490-510 nm; emission: 520$550 \mathrm{~nm}$ ). Membranes were stained with either TMA-DPH (excitation: $340-380 \mathrm{~nm}$; emission: $435-485 \mathrm{~nm}$ ) or FM4-64 (excitation: $510-550 \mathrm{~nm}$; emission: $590 \mathrm{~nm}$ longpass) with exposure times of $200 \mathrm{msec}$. DNA was visualized with DAPI (excitation: 340-380 nm; emission: 435-485 nm) with exposure times of 200 msec. Images were analyzed, adjusted, and cropped using Metamorph software. Higher resolution images can be found in the Supplemental Material.
RNA polymerase stalled elongation complex displacement assays

DNA substrates for RNA polymerase displacement reactions contained the $\lambda_{\mathrm{PR}}$ promoter followed by a 70-bp-long stretch devoid of guanosines in the template strand. Omission of CTP led to the stalling of RNA polymerase at the position of the first template guanosine.

To create the fluorescently labeled DNA substrates, a 433-bp DNA fragment containing the $\lambda_{\text {PR }}$ promoter was PCR-amplified from pJP101 using fluorescently labeled oligonucleotides (oJP036 and oJP037). This PCR product was purified using the Qiagen PCR-cleanup kit. Stalled complexes were prepared in TBF Buffer (40 mM Tris- $\mathrm{HCl}$ at $\mathrm{pH} 7.5,10 \mathrm{mM} \mathrm{MgCl} 2,0.01 \%$ Triton X-100, and $1 \mathrm{mM}$ DTT) by incubating $100 \mathrm{nM}$ E.coli RNA polymerase holoenzyme (Epicentre) and $20 \mathrm{nM}$ fluorescent $433-\mathrm{bp} \lambda_{\mathrm{PR}}$ DNA fragment for $30 \mathrm{~min}$ at $37^{\circ} \mathrm{C}$. The reaction was further incubated for $10 \mathrm{~min}$ at $22^{\circ} \mathrm{C}$ in the presence of 100 $\mathrm{nM}$ nonfluorescent 433-bp $\lambda_{\mathrm{PR}}$ DNA to compete unbound and nonspecifically bound RNA polymerase. To produce stalled elongation complexes, a nucleotide mixture containing GTP, UTP, and ATP at a final concentration of $25 \mu \mathrm{M}$ was incubated with the transcription reaction for $20 \mathrm{~min}$ at $22^{\circ} \mathrm{C}$.

Displacement of stalled RNA polymerase by SpoIIIE was assayed by incubating $10 \mathrm{nM}$ stalled elongation complex with 420 $\mathrm{nM}$ of the SpoIIIE motor domain (SpoIIIE C $_{\text {) }}$ (Ptacin et al. 2008) and $3 \mathrm{mM}$ ATP in SpoIIIE ${ }_{\mathrm{C}}$ reaction buffer $(50 \mathrm{mM}$ Tris- $\mathrm{HCl}$ at $\mathrm{pH} 7.5,10 \mathrm{mM} \mathrm{MgCl} 2$ ). All reactions were incubated for $10 \mathrm{~min}$ at $37^{\circ} \mathrm{C}$ and then stopped by incubation with $0.2 \mathrm{mg} / \mathrm{mL}$ heparin for $5 \mathrm{~min}$ at $22^{\circ} \mathrm{C}$. Reactions were analyzed in $4 \%-20 \%$ precast polyacrylamide gels (Bio-Rad) and run in $1 \times$ TBE $(45 \mathrm{mM}$ Tris-borate at $\mathrm{pH} 8.3,1 \mathrm{mM}$ EDTA) at $20 \mathrm{~mA}$ for $2 \mathrm{~h}$ at $22^{\circ} \mathrm{C}$. Gels were imaged using a Typhoon scanner (GE) and bands quantified using ImageQuant.

To create nonfluorescent stalled elongation complexes with radiolabeled RNA transcripts, a 278-bp DNA fragment containing $\lambda_{\mathrm{PR}}$ was PCR-amplified from pJP101 (oligonucleotide primers oJP033 and oJP034) and purified using the Qiagen PCRcleanup kit. Stalled complexes were prepared in TBF Buffer by incubating $50 \mathrm{nM}$ RNA polymerase and $100 \mathrm{nM}$ DNA template for $30 \mathrm{~min}$ at $37^{\circ} \mathrm{C}$. To produce stalled elongation complexes, the mixture was incubated with GTP, UTP, and ATP at final concentrations of $2.5 \mu \mathrm{M}$, and $0.17 \mu \mathrm{M} \alpha^{32} \mathrm{P}$-GTP for $20 \mathrm{~min}$ at $22^{\circ} \mathrm{C}$.

Displacement of radiolabeled complexes by SpoIIIE was assayed by incubating $5 \mathrm{nM}$ radioactive stalled elongation complex with $200 \mathrm{nM}$ SpoIIIE $\mathrm{C}_{\mathrm{C}} 3 \mathrm{mM}$ ATP, and $25 \mu \mathrm{M}$ unlabeled GTP in SpoIIIE $E_{C}$ reaction buffer for $10 \mathrm{~min}$ at $37^{\circ} \mathrm{C}$. Unlabeled GTP was added to the reaction to prevent the formation of new radiolabeled stalled complexes resulting from the reassociation of RNA polymerase with $\lambda_{\mathrm{PR}}$ DNA after displacement. Reactions treated with NspI were performed in $1 \times$ NEB2 buffer with $1 \mathrm{mg} / \mathrm{mL}$ BSA and 0.025 units of NspI per microliter. All reactions were incubated for $10 \mathrm{~min}$ at $37^{\circ} \mathrm{C}$ and then stopped by incubation with $0.2 \mathrm{mg} / \mathrm{mL}$ heparin for $5 \mathrm{~min}$ at $22^{\circ} \mathrm{C}$. Gels were run as described above, exposed on phosphorimager screens (Amersham Biosciences), and imaged using a Typhoon scanner.

\section{Plasmid construction}

pKM193 [pelB:: $P_{\text {spoIID }}$-tetR-gfp (tet)] was generated in a threeway ligation with a HindIII-BamHI PCR product containing tetR-gfp and an optimized ribosome-binding site (Vellanoweth and Rabinowitz 1992) (oligonucleotide primers oDR78 and oDR188 with pBW3 [Dworkin and Losick 2002] as template) 
and an EcoRI-HindIII $\mathrm{P}_{\text {spoIID }}$ promoter fragment from pDR78 (Rudner and Losick 2002) and pKM033 (pelB::tet) cut with EcoRI and BamHI. pKM033 is an ectopic integration vector for double cross-over integrations at the nonessential pelB locus (K.A. Marquis and D.Z. Rudner, unpubl.).

pKM219 $\left[y k p A B\left(130^{\circ}\right)(\text { tetO })_{120}\right.$ (spec)] was generated by inserting a PCR product containing the $(\text { tet } O)_{120}$ array (oligonucleotide primers oDR458 and oDR459 with pLAU44 [Lau et al. 2003] as template) into pKM210 between HindIII and XhoI. pKM210 was generated by inserting a 600-bp PCR product containing part of $y k p A$ and $y k p B$ (oligonucleotide primers oDR494 and oDR495 with PY79 genomic DNA as template) into pUS19 (pUC19-spec) between EcoRI and BamHI. pKM210 is a single cross-over integration vector for insertions at $130^{\circ}$ (K.A. Marquis and D.Z. Rudner, unpubl.).

pJP101 [ $\lambda_{\text {PR }}$ promoter] was generated by inserting a PCR product containing the $\lambda_{\mathrm{PR}}$ promoter (oligonucleotide primer oJP033 and oJP034 with pPIA2-6 as template [Davenport et al. 2000]) into the SacII site of pJB103 (Bath et al. 2000).

\section{Acknowledgments}

We thank members of the Rudner laboratory past and present, Tom Rapoport, Richard Losick, Antoine Van Oijen, and Ann Hochschild for valuable discussions. We acknowledge Masaya Fujita, Alan Grossman, and David Sherratt for strains and plasmids and Remi Bernhard for help with microscopy. This project was initiated by D.Z.R. and S.B. when they were postdoctoral fellows in the laboratory of R. Losick, and these authors wish to acknowledge his enduring mentorship. This paper is dedicated to Nick Cozzarelli. Support for this work comes from the National Institute of Health Grant GM073831-01A1 and the Hellman Family Faculty Fund. D.Z.R. is supported by the Damon Runyon Cancer Research Foundation (DRS 44-05). B.M.B was supported in part by the Damon Runyon Cancer Research Foundation (DRG 1804-04) and by the Charles A. King Trust, Bank of America, Co-Trustee. M.N. was supported by the Human Frontier Science Program.

\section{References}

Andrews, C. and Richardson, J.P. 1985. Transcription termination factor $\rho$ mediates simultaneous release of RNA transcripts and DNA template from complexes with Escherichia coli RNA polymerase. J. Biol. Chem. 260: 5826-5831.

Aussel, L., Barre, F.X., Aroyo, M., Stasiak, A., Stasiak, A.Z., and Sherratt, D. 2002. FtsK is a DNA motor protein that activates chromosome dimer resolution by switching the catalytic state of the XerC and XerD recombinases. Cell 108: 195-205.

Bath, J., Wu, L.J., Errington, J., and Wang, J.C. 2000. Role of Bacillus subtilis SpoIIIE in DNA transport across the mother cell-prespore division septum. Science 290: 995-997.

Ben-Yehuda, S., Rudner, D.Z., and Losick, R. 2003a. Assembly of the SpoIIIE DNA translocase depends on chromosome trapping in Bacillus subtilis. Curr. Biol. 13: 2196-2200.

Ben-Yehuda, S., Rudner, D.Z., and Losick, R. 2003b. RacA, a bacterial protein that anchors chromosomes to the cell poles. Science 299: 532-536.

Bigot, S., Saleh, O.A., Lesterlin, C., Pages, C., El Karoui, M., Dennis, C., Grigoriev, M., Allemand, J.F., Barre, F.X., and Cornet, F. 2005. KOPS: DNA motifs that control E. coli chromosome segregation by orienting the FtsK translocase. EMBO J. 24: 3770-3780.
Bogush, M., Xenopoulos, P., and Piggot, P.J. 2007. Separation of chromosome termini during sporulation of Bacillus subtilis depends on SpoIIIE. J. Bacteriol. 189: 3564-3572.

Bolon, D.N., Grant, R.A., Baker, T.A., and Sauer, R.T. 2004. Nucleotide-dependent substrate handoff from the SspB adaptor to the AAA+ ClpXP protease. Mol. Cell 16: 343-350.

Brewer, B.J. 1988. When polymerases collide: Replication and the transcriptional organization of the E. coli chromosome. Cell 53: 679-686.

Britton, R.A. and Grossman, A.D. 1999. Synthetic lethal phenotypes caused by mutations affecting chromosome partitioning in Bacillus subtilis. J. Bacteriol. 181: 5860-5864.

Burton, B.M., Marquis, K.A., Sullivan, N.L., Rapoport, T.A., and Rudner, D.Z. 2007. The ATPase SpoIIIE transports DNA across fused septal membranes during sporulation in Bacillus subtilis. Cell 131: 1301-1312.

Crampton, D.J., Ohi, M., Qimron, U., Walz, T., and Richardson, C.C. 2006. Oligomeric states of bacteriophage T7 gene 4 primase/helicase. J. Mol. Biol. 360: 667-677.

Davenport, R.J., Wuite, G.J., Landick, R., and Bustamante, C. 2000. Single-molecule study of transcriptional pausing and arrest by E. coli RNA polymerase. Science 287: 2497-2500.

Doan, T., Marquis, K.A., and Rudner, D.Z. 2005. Subcellular localization of a sporulation membrane protein is achieved through a network of interactions along and across the septum. Mol. Microbiol. 55: 1767-1781.

Dworkin, J. and Losick, R. 2002. Does RNA polymerase help drive chromosome segregation in bacteria? Proc. Natl. Acad. Sci. 99: 14089-14094.

Errington, J. 2003. Regulation of endospore formation in Bacillus subtilis. Nat. Rev. Microbiol. 1: 117-126.

Errington, J., Bath, J., and Wu, L.J. 2001. DNA transport in bacteria. Nat. Rev. Mol. Cell Biol. 2: 538-545.

French, S. 1992. Consequences of replication fork movement through transcription units in vivo. Science 258: 1362-1365.

Fujita, M. and Losick, R. 2003. The master regulator for entry into sporulation in Bacillus subtilis becomes a cell-specific transcription factor after asymmetric division. Genes \& Dev. 17: 1166-1174.

Grigorova, I.L., Phleger, N.J., Mutalik, V.K., and Gross, C.A. 2006. Insights into transcriptional regulation and $\sigma$ competition from an equilibrium model of RNA polymerase binding to DNA. Proc. Natl. Acad. Sci. 103: 5332-5337.

Harwood, C.R. and Cutting, S.M. 1990. Molecular biological methods for Bacillus. Wiley, New York.

Hilbert, D.W. and Piggot, P.J. 2004. Compartmentalization of gene expression during Bacillus subtilis spore formation. Microbiol. Mol. Biol. Rev. 68: 234-262.

Ju, J., Mitchell, T., Peters 3rd, H., and Haldenwang, W.G. 1999. $\sigma$ Factor displacement from RNA polymerase during Bacillus subtilis sporulation. J. Bacteriol. 181: 4969-4977.

Kohler, P. and Marahiel, M.A. 1997. Association of the histonelike protein HBsu with the nucleoid of Bacillus subtilis. I. Bacteriol. 179: 2060-2064.

Lau, I.F., Filipe, S.R., Soballe, B., Okstad, O.A., Barre, F.X., and Sherratt, D.J. 2003. Spatial and temporal organization of replicating Escherichia coli chromosomes. Mol. Microbiol. 49: 731-743.

Levy, O., Ptacin, J.L., Pease, P.J., Gore, J., Eisen, M.B., Bustamante, C., and Cozzarelli, N.R. 2005. Identification of oligonucleotide sequences that direct the movement of the Escherichia coli FtsK translocase. Proc. Natl. Acad. Sci. 102: 17618-17623.

Li, Z. and Piggot, P.J. 2001. Development of a two-part transcription probe to determine the completeness of temporal and spatial compartmentalization of gene expression during 
bacterial development. Proc. Natl. Acad. Sci. 98: 1253812543.

Lin, D.C., Levin, P.A., and Grossman, A.D. 1997. Bipolar localization of a chromosome partition protein in Bacillus subtilis. Proc. Natl. Acad. Sci. 94: 4721-4726.

Lord, M., Barilla, D., and Yudkin, M.D. 1999. Replacement of vegetative $\sigma \mathrm{A}$ by sporulation-specific $\sigma \mathrm{F}$ as a component of the RNA polymerase holoenzyme in sporulating Bacillus subtilis. J. Bacteriol. 181: 2346-2350.

Massey, T.H., Mercogliano, C.P., Yates, J., Sherratt, D.J., and Lowe, J. 2006. Double-stranded DNA translocation: Structure and mechanism of hexameric FtsK. Mol. Cell 23: 457469.

Michaelis, C., Ciosk, R., and Nasmyth, K. 1997. Cohesins: Chromosomal proteins that prevent premature separation of sister chromatids. Cell 91: 35-45.

Pease, P.J., Levy, O., Cost, G.J., Gore, J., Ptacin, J.L., Sherratt, D., Bustamante, C., and Cozzarelli, N.R. 2005. Sequence-directed DNA translocation by purified FtsK. Science 307: 586-590.

Pogliano, K., Harry, E., and Losick, R. 1995. Visualization of the subcellular location of sporulation proteins in Bacillus subtilis using immunofluorescence microscopy. Mol. Microbiol. 18: 459-470.

Possoz, C., Filipe, S.R., Grainge, I., and Sherratt, D.J. 2006. Tracking of controlled Escherichia coli replication fork stalling and restart at repressor-bound DNA in vivo. EMBO J. 25: 2596-2604

Ptacin, J.L., Nollmann, M., Becker, E.C., Cozzarelli, N.R., Pogliano, K., and Bustamante, C. 2008. Sequence-directed DNA export guides chromosome translocation during sporulation in Bacillus subtilis. Nat. Struct. Mol. Biol. 15: 485-493.

Rocha, E.P. 2004. The replication-related organization of bacterial genomes. Microbiol. 150: 1609-1627.

Rudner, D.Z. and Losick, R. 2001. Morphological coupling in development: Lessons from prokaryotes. Dev. Cell 1: 733-742.

Rudner, D.Z. and Losick, R. 2002. A sporulation membrane protein tethers the pro- $\sigma \mathrm{K}$ processing enzyme to its inhibitor and dictates its subcellular localization. Genes \& Dev. 16: $1007-1018$.

Ryter, A., Schaeffer, P., and Ionesco, H. 1966. [Cytologic classification, by their blockage stage, of sporulation mutants of Bacillus subtilis Marburg]. Ann. Inst. Pasteur (Paris) 110: 305-315.

Saleh, O.A., Perals, C., Barre, F.X., and Allemand, J.F. 2004. Fast, DNA-sequence independent translocation by $\mathrm{FtsK}$ in a single-molecule experiment. EMBO J. 23: 2430-2439.

Selby, C.P. and Sancar, A. 1993. Molecular mechanism of transcription-repair coupling. Science 260: 53-58.

Setlow, P. 1995. Mechanisms for the prevention of damage to DNA in spores of Bacillus species. Annu. Rev. Microbiol. 49: 29-54.

Sharpe, M.E. and Errington, J. 1995. Postseptational chromosome partitioning in bacteria. Proc. Natl. Acad. Sci. 92: 8630-8634.

Sharp, M.D. and Pogliano, K. 1999. An in vivo membrane fusion assay implicates SpoIIIE in the final stages of engulfment during Bacillus subtilis sporulation. Proc. Natl. Acad. Sci. 96: $14553-14558$.

Stragier, P. and Losick, R. 1996. Molecular genetics of sporulation in Bacillus subtilis. Annu. Rev. Genet. 30: 297-341.

Vellanoweth, R.L. and Rabinowitz, J.C. 1992. The influence of ribosome-binding-site elements on translational efficiency in Bacillus subtilis and Escherichia coli in vivo. Mol. Microbiol. 6: 1105-1114.

Viollier, P.H., Thanbichler, M., McGrath, P.T., West, L., Mee- wan, M., McAdams, H.H., and Shapiro, L. 2004. Rapid and sequential movement of individual chromosomal loci to specific subcellular locations during bacterial DNA replication. Proc. Natl. Acad. Sci. 101: 9257-9262.

Wang, X., Possoz, C., and Sherratt, D.J. 2005. Dancing around the divisome: Asymmetric chromosome segregation in Escherichia coli. Genes \& Dev. 19: 2367-2377.

Webb, C.D., Teleman, A., Gordon, S., Straight, A., Belmont, A., Lin, D.C., Grossman, A.D., Wright, A., and Losick, R. 1997. Bipolar localization of the replication origin regions of chromosomes in vegetative and sporulating cells of $B$. subtilis. Cell 88: 667-674.

Wu, L.J. and Errington, J. 1994. Bacillus subtilis spoIIIE protein required for DNA segregation during asymmetric cell division. Science 264: 572-575.

$\mathrm{Wu}$, L.J. and Errington, J. 1997. Septal localization of the SpoIIIE chromosome partitioning protein in Bacillus subtilis. EMBO J. 16: 2161-2169.

Wu, L.J. and Errington, J. 1998. Use of asymmetric cell division and spoIIIE mutants to probe chromosome orientation and organization in Bacillus subtilis. Mol. Microbiol. 27: 777786.

Wuite, G.J., Smith, S.B., Young, M., Keller, D., and Bustamante, C. 2000. Single-molecule studies of the effect of template tension on T7 DNA polymerase activity. Nature 404: 103106.

Youngman, P.J., Perkins, J.B., and Losick, R. 1983. Genetic transposition and insertional mutagenesis in Bacillus subtilis with Streptococcus faecalis transposon Tn917. Proc. Nat1. Acad. Sci. 80: 2305-2309.

Zhang, L., Higgins, M.L., Piggot, P.J., and Karow, M.L. 1996. Analysis of the role of prespore gene expression in the compartmentalization of mother cell-specific gene expression during sporulation of Bacillus subtilis. I. Bacteriol. 178: 2813-2817.

Zupancic, M.L., Tran, H., and Hofmeister, A.E. 2001. Chromosomal organization governs the timing of cell type-specific gene expression required for spore formation in Bacillus subtilis. Mol. Microbiol. 39: 1471-1481. 


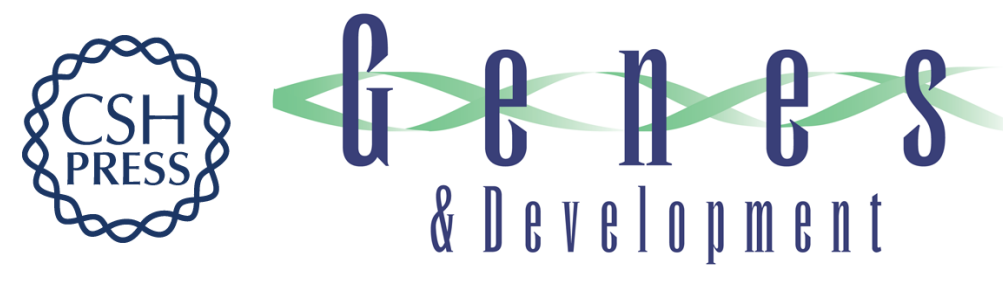

\section{SpollIE strips proteins off the DNA during chromosome translocation}

Kathleen A. Marquis, Briana M. Burton, Marcelo Nollmann, et al.

Genes Dev. 2008, 22:

Access the most recent version at doi:10.1101/gad.1684008

Supplemental

Material

References

This article cites 55 articles, 30 of which can be accessed free at:

http://genesdev.cshlp.org/content/22/13/1786.full.html\#ref-list-1

\section{License}

Email Alerting Service

http://genesdev.cshlp.org/content/suppl/2008/07/02/22.13.1786.DC1 right corner of the article or click here.

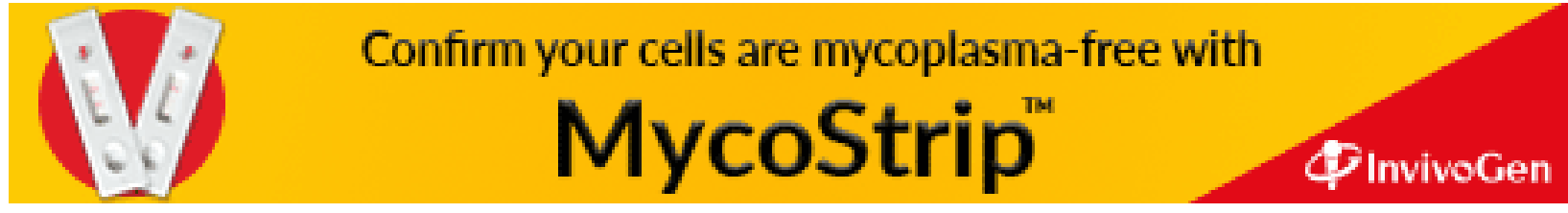

